\title{
Modeling of the pyrolysis of plywood exposed to heat fluxes under cone calorimeter
}

\author{
TALAL FATEH, FRANCK RICHARD, and THOMAS ROGAUME \\ Institut Pprime / Département FTC \\ Téléport 2 - 1, avenue Clément Ader, 86961 Futuroscope, France
}

\begin{abstract}
In this paper, the thermal decomposition of plywood is investigated based on the solid mass loss rate (MLR) modeling. The multi-scale approach followed here allows first to establish, at a small scale, the kinetic mechanism during the solid thermal decomposition and then validate it at a larger scale.

At small scale, experiments were conducted by using Thermo-gravimetric analysis (TGA) coupled to gas analysis with the FTIR technique under nitrogen and air atmospheres for five heating rates.

Thermo-gravimetric results were also used to propose a kinetic mechanism for the thermal decomposition of the sample. The kinetic parameters of the different identified reactions were estimated by using an optimization technique, namely the Genetic Algorithms (GA) method. The mass loss rate model predictions show a good agreement with the experimental data.
\end{abstract}

At a larger scale, experiments were carried out in a cone calorimeter coupled to FTIR gas analysis under air atmosphere. The pyrolysis model developed at the TGA scale was used in numerical simulations of cone calorimeter experiments taking into account the heat transfer modeling into the sample. The thermal properties (e.g. thermal conductivity and specific heat capacity) of the condensed phase species (which are products given by the thermal decomposition of the virgin material) identified at the TGA scale were estimated for an incident heat flux of $30 \mathrm{~kW} \cdot \mathrm{m}^{-2}$ with the same optimization technique used at the small scale: the GA method.

The same work has also been conducted with a simpler well known pyrolysis model developed for charring materials. The results have been compared to those of the first mechanism in order to show the influence of the complexity of the model on the prediction of the thermal decomposition of plywood. The heat transfer model was kept the same for both pyrolysis models. Only the number of identified condensed phase reactions and species is different. The detailed mechanism ( 5 steps) gives better results than the simpler one (3steps) concerning the mass loss rate prediction and worse results for the temperature prediction of the back surface of the sample. However the 3 steps model gives unrealistic results concerning the prediction of the condensed phase species mass fractions. In fact, the 3 steps model predicts that plywood is not fully burned at the end of the test (for $30 \mathrm{~kW} . \mathrm{m}^{-2}$ ) which was not observed in the experiment.

KEYWORDS: thermal degradation, cone calorimeter, TGA, GPYRO, plywood, pyrolysis model, genetic algorithm. 


\section{NOMENCLATURE LISTING}

\begin{tabular}{llll}
$A$ & Pre-exponential factor $\left(\mathrm{s}^{-1}\right)$ & Greek \\
$c_{p}$ & Specific heat capacity $\left(\mathrm{j} \cdot \mathrm{kg}^{-1} \cdot \mathrm{K}\right)$ & $\alpha$ Conversion factor $(-)$ \\
$E$ & Activation of energy $\left(\mathrm{J} \cdot \mathrm{mol}^{-1}\right)$ & $\beta$ Heating rate $\left(\mathrm{K} \cdot \mathrm{min}^{-1}\right)$ \\
$h$ & Heat transfer coefficient $\left(\mathrm{W} \cdot \mathrm{m}^{-2} \cdot \mathrm{K}\right)$ & $k$ Thermal conductivity $\left(\mathrm{W} \cdot \mathrm{m}^{-1} \cdot \mathrm{K}\right)$ \\
$m$ & Mass $(\mathrm{kg})$ & $\varepsilon$ & Emissivity $(-)$ \\
$n$ & Reaction order & $\rho$ Density $\left(\mathrm{kg} \cdot \mathrm{m}^{-3}\right)$ \\
$q$ & Heat flux $\left(\mathrm{kW} \cdot \mathrm{m}^{-2}\right)$ & $\omega$ Reaction rate $\left(\mathrm{kg} \cdot \mathrm{m}^{-3} \cdot \mathrm{s}^{-1}\right)$ \\
$w$ & Reaction rate $\left(\mathrm{kg} \cdot \mathrm{m}^{-3} \cdot \mathrm{s}^{-1}\right)$ & $v$ & Stoechiometric coefficient $(-)$ \\
$T$ & Temperature $(\mathrm{K})$ & $v$ Thermal diffusivity $\left(\mathrm{m}^{2} \cdot \mathrm{s}^{-1}\right)$ \\
$T_{o}$ & Ambient temperature $(\mathrm{K})$ & Subscripts \\
$t$ & Time $(\mathrm{s})$ & $G A$ & Genetic Algorithm \\
$\mathrm{Z}$ & Thickness $(\mathrm{m})$ & 0 & Mass loss rate \\
$\mathrm{w}$ & Rate of conversion $\left(\mathrm{s}^{-1}\right)$ & $f$ & Final condition \\
$\Delta \mathrm{H}$ & Change in enthalpy $\left(\mathrm{J} \cdot \mathrm{kg}^{-1}\right)$ & $i$ & Species number \\
$\mathrm{y}$ & Mass fraction of species & $k$ & Reaction number \\
$\mathrm{R}$ & Universal gas constant $\left(\mathrm{J}^{-1} \mathrm{~mol}^{-1} \cdot \mathrm{K}^{-1}\right)$ & $k$ & \\
$\Delta \mathrm{z}$ & Size of grid cell $(\mathrm{m})$ & \multicolumn{2}{l}{}
\end{tabular}

\section{INTRODUCTION}

It is required for fire safety purposes to be able to predict the behavior of the new construction materials in fires by determining some parameters such as the kinetic parameters and the thermal properties at laboratory scale [1].

Thermo-gravimetric analysis is a traditional method to investigate thermal decomposition by determination of the weight loss characteristics for different conditions of heating rate and atmosphere [1-7].

By the coupling to Fourier transformed infrared spectroscopy (TGA-FTIR), thermo-gravimetric analysis is used in order to investigate the chemical reactions occurring during the thermal decomposition. This technique is based on the comparison of the gas emissions to the mass evolution in controlled conditions [7]. The advantage of the TGA is that the conditions of thermal decomposition are well known and controlled due to the small mass degraded and the slow heating rate conditions. Most of the TGA experiments have been conducted under low heating rates (less than $50 \mathrm{~K} \cdot \mathrm{min}^{-1}$ ) to allow the separation and visualization of all reaction steps occurring during the thermal decomposition. Unfortunately, TGA analyses do not fully represent real fire conditions. The heating rates are low compared to the heat fluxes occurring in real fires. Furthermore, temperature and mass gradients into the solid are not examined in TGA analyses [2].

In contrast, the cone calorimeter is a standard apparatus for testing of materials [8]. It provides the thermal properties that could predict the behavior of materials at larger scales. The conditions of thermal decomposition are more realistic. They include thick materials, large heat fluxes and an analysis of the heat and mass transfer phenomena. However, many parameters are required for the development of pyrolysis models. Indeed in addition to the kinetic parameters associated to reactions occurring in condensed phase, the thermal properties associated to heat and mass transfer phenomena into the solid have also to be determined. Actually, many numerical codes are developed to simulate the decomposition of solid fuels at large scales (as in a cone calorimeter) like GPYRO [9-12]. The calculations take into account heat and mass transfer phenomena into the solid as well as the kinetics of thermal decomposition within the solid. 
Some of these parameters cannot be measured directly from experiments and must be estimated by using optimization techniques like the Genetic Algorithms (GA) method. [12-16].

In this paper, the thermal decomposition of plywood is investigated based on the solid mass loss rate (MLR) modeling. At small scale, experiments were conducted by using Thermo-gravimetric analysis coupled to gas analysis with FTIR technique under nitrogen and air atmospheres for five heating rates. Thermo-gravimetric results were used to propose a kinetic mechanism for the thermal decomposition of the solid. The kinetic parameters were estimated by using the GA method. At larger scale, experiments were carried out in cone calorimeter coupled to FTIR gas analysis under air. At this scale, heat and mass transfer phenomena into the sample are taking into account and thermal properties of the condensed phase species related to reactions have been estimated by the GA method. The influence of the kinetic model (in terms of number of condensed phase species and reactions) on the MLR prediction is examined. The same methodology was used for the temperature and composition evolution of the solid.

\section{RESULTS AND DISCUSSION}

Wood is considered to be one of the oldest and most important materials in construction. For instance, plywood (i.e. wood panel made of thin sheets of wood veneer) is one of the most widely used wood products in green construction.

In this paper, a plywood mainly made of oakum classed B in the euro-class code was used. The elementary analysis shows that the plywood is mainly constituted of $42.6 \%$ wt. of carbon, $43.9 \%$ wt. of oxygen, 5.5 $\%$ wt. of hydrogen and $1.9 \%$ wt. of nitrogen.

For the first step considering the thermal decomposition of the sample in TGA, a $5 \mathrm{mg}$ sample of powdered plywood was initially placed in the sample pan and then heated from room temperature (about $20{ }^{\circ} \mathrm{C}$ ) to $1000{ }^{\circ} \mathrm{C}$ at different heating rates: $10,15,20,30$ and $40{ }^{\circ} \mathrm{C} \min ^{-1}$. The furnace was flushed with nitrogen or air flow rate (depending of the thermal atmosphere studied) at $90 \mathrm{~mL} \cdot \mathrm{min}^{-1}$ via the transport gas inlet to maintain an inert or oxidative atmosphere during the thermal decomposition process of the sample. Products released during the thermal degradation of plywood were analyzed with a FTIR spectrometer placed at the outlet of TGA device. Transfer lines between the two apparatus as well as the FTIR gas measurement cell were heated at $230{ }^{\circ} \mathrm{C}$ to avoid condensation and water-soluble compounds trapping. IR spectra were recorded in the spectral range of $4000-650 \mathrm{~cm}^{-1}$ with a $4 \mathrm{~cm}^{-1}$ resolution and 16 scans.

Figure 1 presents the mass loss rate (MLR) as function of temperature at different heating rates in inert and air atmospheres respectively.

Thermal degradation begins at $200{ }^{\circ} \mathrm{C}$ independently of the heating rate. An increase of the heating rate leads to a shift of the MLR curves towards the higher temperatures without changing the temperature at which mass loss starts taking place [19]. The evolutions of the mass loss rates are also qualitatively similar for all the heating rates. The chemical kinetic of the thermal decomposition seems to be independent of the heating rate chosen here.

The kinetic mechanism of the thermal decomposition of the plywood has been investigated in details in a previous work [12]. The mechanism of thermal decomposition was proposed from the coupled analysis of the curves of mass loss and mass loss rate.

The analysis was performed by examining the change in slope of the curves and the corresponding gaseous emissions. Several reaction steps were identified (all details are available in [12]). The mechanism can be written as follows:

$$
\text { Plywood } \underset{N_{2}}{\longrightarrow} \text { oPlywood } \underset{N_{2}}{\longrightarrow} \text { BPlywood } \underset{N_{2}}{\longrightarrow} \text { oPlywood } \stackrel{\text { Air }}{\longrightarrow} \text { APlywood } \stackrel{\text { Air }}{\longrightarrow} \text { APlywood }
$$

where $\alpha, \beta, \sigma, \theta$ and $\lambda$ plywood are related to the different condensed phase species identified. 


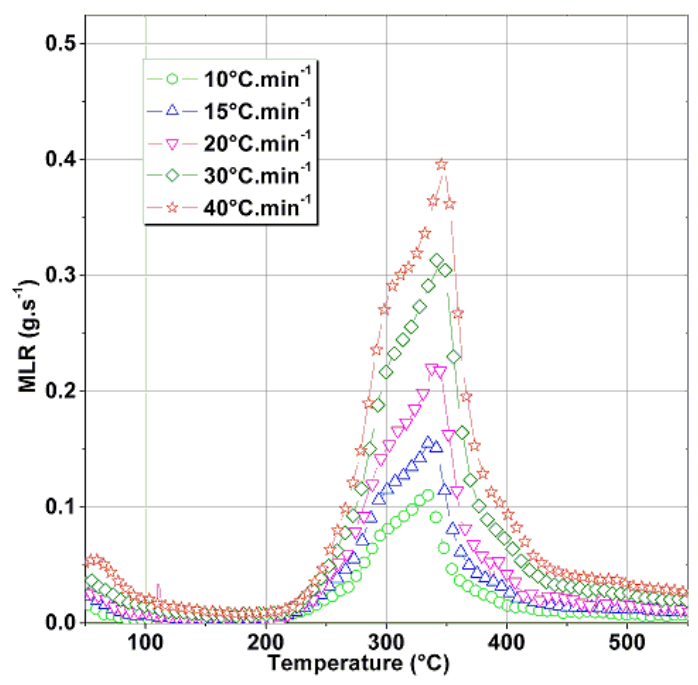

Under nitrogen

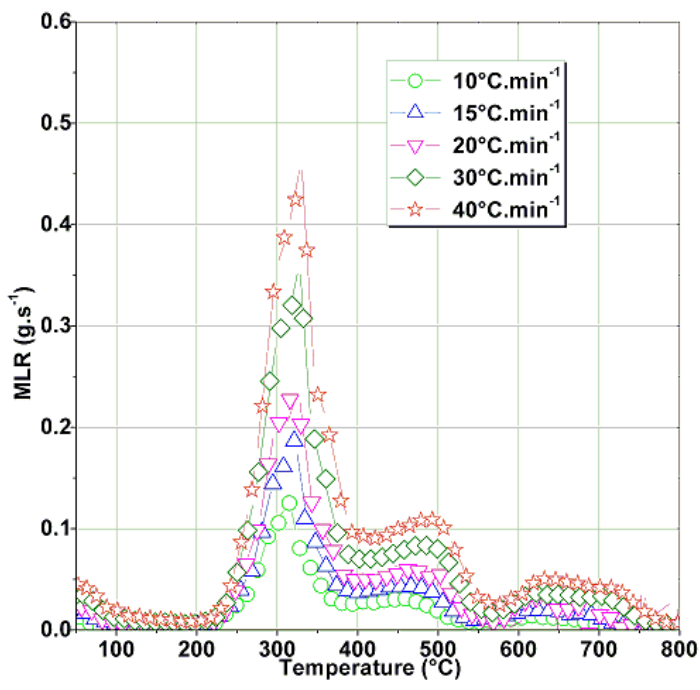

Under air

Fig. 1. Experimental curves of MLR under nitrogen and air.

The model used to describe the thermal decomposition of the solid according to the chemical mechanism established before is based on the degree of conversion $(\alpha)$ of the different condensed phase species identified during the decomposition of the sample [9-12, 14-15]:

$\alpha=\frac{m_{0}-m_{t}}{m_{0}-m_{f}}$

where $m_{0}$ is the initial mass of the sample, $m_{t}$ is the sample mass at the time $\mathrm{t}$ and $m_{f}$ is the sample mass remained at the end of the test.

The rate of conversion of each species is assumed to be described by the product of the rate constant, $k(\mathrm{~T})$ and the differential conversion function, $f(\alpha)$ as shown in the following expression:

$\frac{d \alpha_{i, k}}{d t}=w_{k}=k(T) f(\alpha)=A_{k} \exp \left(-\frac{E_{k}}{R T}\right)\left(1-\alpha_{k}\right)^{n_{k}} y_{O_{2}}^{\delta}$

The rate constant is an Arrhenius equation and the conversion function is the reaction model. The reaction model is taking as a "n-order" model where n depends on the reaction considered. $\delta$ is the Kronecker symbol.

The mass fraction of $\mathrm{O}_{2}$ is only considered for oxidative reactions, it is not considered for pure pyrolysis reactions.

The set of ordinary differential equations related to the chemical mechanism established before is written as the following: 


$$
\begin{cases}\frac{d}{d t} \text { plywood }=-w_{1} & \frac{d}{d t} \sigma_{\text {plywood }}=v_{3} w_{3}-w_{4} \\ \frac{d}{d t} \alpha_{\text {plywood }}=v_{1} w_{1}-w_{2} & \frac{d}{d t} \theta_{\text {plywood }}=v_{4} w_{4}-w_{5} \\ \frac{d}{d t} \beta_{\text {plywood }}=v_{2} w_{2}-w_{3} & \frac{d}{d t} \lambda_{\text {plywood }}=v_{5} w_{5}\end{cases}
$$

The unknown parameters are estimated by using a model fitting method. The principle is to find the set of parameters that best fits TGA non isothermal experimental mass loss rate curves by using the model described before. The optimization method used here is the Genetic Algorithms method.

We conclude before that the heating rate does not influence the kinetics of thermal decomposition. The estimation of the best set of parameters is made for all heating rates (i.e. only one set of parameters is found for all the heating rates considered here). The values of the kinetic parameters are presented in Table 1.

Table 1. Estimated kinetic parameters.

\begin{tabular}{|l|l|c|c|c|c|c|}
\hline \multicolumn{1}{|c|}{ Reactant } & Product & $\mathbf{L o g}_{\mathbf{1 0}} \mathbf{A} \mathbf{( s}^{\mathbf{- 1}} \mathbf{)}$ & $\mathbf{E ~ ( \mathbf { k J . m o l } ^ { - 1 } )}$ & $\mathbf{n ~ ( - )}$ & $\mathbf{v ~ ( - )}$ & $\Delta \mathbf{H}\left(\mathbf{J J g}^{-\mathbf{1}}\right)$ \\
\hline Plywood & $\alpha$ Plywood & 8.16 & 108.60 & 1.07 & 0.74 & $1.86 \times 10^{1}$ \\
\hline$\alpha$ Plywood & $\beta$ Plywood & 7.04 & 96.50 & 1.02 & 0.84 & $1.20 \times 10^{2}$ \\
\hline$\beta$ Plywood & $\sigma$ Plywood & 5.42 & 79.76 & 3.09 & 0.51 & $7.41 \times 10^{1}$ \\
\hline$\sigma$ Plywood & $\theta$ Plywood & 6.87 & 111.50 & 1.35 & 0.50 & $-1.12 \times 10^{5}$ \\
\hline$\theta$ Plywood & $\lambda$ plywood & 5.15 & 113.90 & 1.39 & 0.40 & $-5.50 \times 10^{3}$ \\
\hline
\end{tabular}

Figure 2 presents the simulated mass loss rates at different heating rates in inert and air atmospheres respectively. The predicted MLR evolutions show a good agreement with the experimental results. We can conclude at this step that the hypothesis of independence between the heating rate and the kinetics of the condensed phase is validated and so is the model.

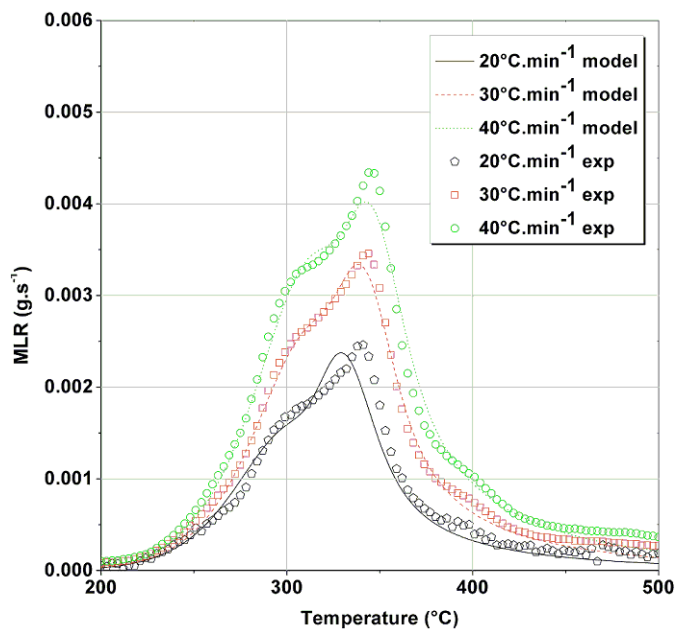

Under nitrogen

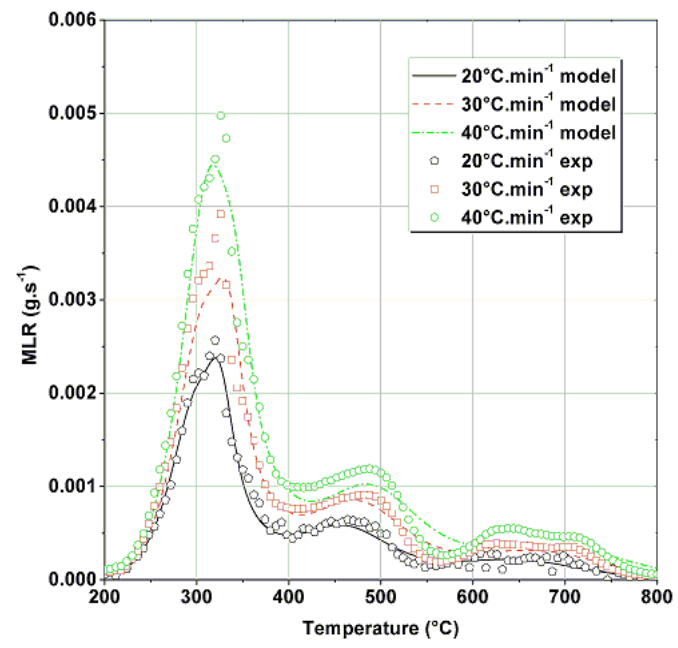

Under air

Fig. 2 Calculated MLR under nitrogen and air atmospheres. 
TGA experiments are far from conditions encountered in real fires. Indeed, during this type of experiments heat and mass transfer into the sample are supposed to be controlled.

By using a very small size of samples, a zero gradient for temperature and gaseous concentrations into the sample is assumed. The temperature and gaseous concentrations into the sample are assumed to be those of the furnace.

At this step, the kinetic part of the pyrolysis model is developed. Reactions occurring in condensed phase have been identified and associated parameters have been estimated. Before applying the model in real fire conditions, the thermal part of the model has to be developed. However, in order to consider heat transfer, thermal properties of condensed phase species involved in the reactions must be known.

The estimation of the thermal properties of the different condensed phase species was carried out using a cone calorimeter apparatus (ISO 5660) [8]. This larger scale allows considering the heat transfer into the solid by burning thermally thick materials. The measurements are conducted by putting the sample holder in horizontal position under ambient air atmosphere with well-ventilated conditions.

The heat fluxes studied were 20,30, 40, 50, 60 and $70 \mathrm{~kW} \cdot \mathrm{m}^{-2}$. Experiments were realized with a piloted ignition.

The sample size was $100 \times 100 \times 18 \mathrm{~mm}^{3}$ and the mass of samples was about $95 \mathrm{~g}$. The sample was insulated at its back surface using a $46 \mathrm{~mm}$ thick ceramic paper in order to reduce heat losses as prescribed in the ISO 5660 standard [8].

The tests were stopped $32 \mathrm{~min}$ after ignition or after $30 \mathrm{~min}$ if no ignition has occurred. The mass loss, mass loss rate and ignition time were recorded during the tests.

Figure 3 presents the evolutions of mass loss and mass loss rates respectively as a function of time obtained in the cone calorimeter under different heat flux values. We can notice that an increase in the heat flux corresponds to a decrease in the delay necessary to lose the same quantity of mass. It should be noted that the final weight of the sample depends on the incident heat flux.
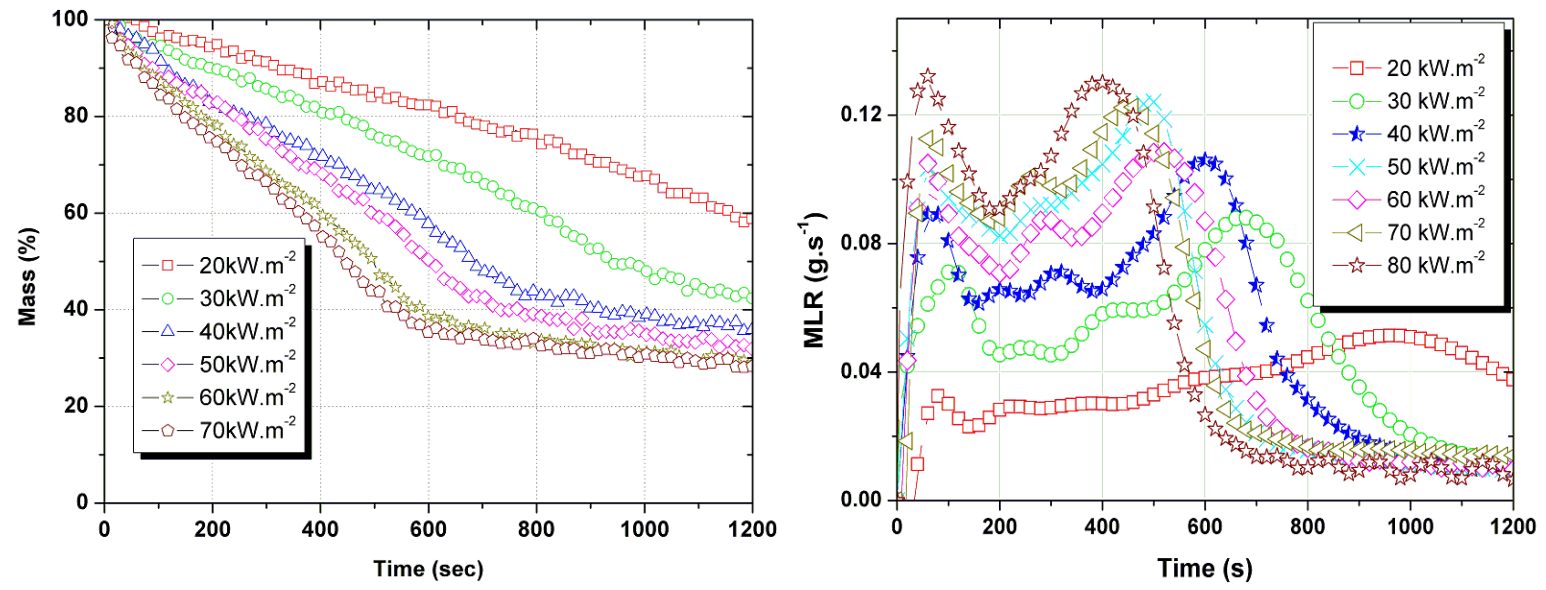

Fig. 3. Evolutions of mass and mass loss rate as a function of time at different heat fluxes.

To capture more details about the behavior of the material [11], the mass loss rate of the sample in function of time could be analyzed.

The shapes of the mass loss rate curves are similar for all heat fluxes. The different peaks could be attributed to the degradation of the different layers of plywood excepted for the last one that could be attributed to the heat feedback due to the back surface insulation of the sample. This experimental bias is less pronounced for the lower heat fluxes. Indeed at the time where the temperature of the solid growth up 
by volume due to the insulation of the back surface, the remaining mass to burn is lower for the low heat fluxes and the remaining mass to burn is a key parameter affecting the mass loss rate.

At this scale (large scale in cone calorimeter), numerical simulations have been conducted using the GPYRO code [9] which includes heat and mass transfer phenomena in addition to the kinetic description of the solid decomposition developed before at the TGA scale.

The material used here is a charring material. For this type of material, the primary fuel generation zone can be well localized below the surface, at a reaction front that separates the char layer from the virgin layer. It is the reason why the MLR decreases after the first high peak (Fig 3). The char layer at the surface of the sample acts as a thermal barrier.

The transient mass loss rate is due to several phenomena like heat transfer and the kinetics of decomposition during the process that governs the release rate of gases.

It is the reason why all of these processes are taking into account in GPYRO code. Here we consider a porous solid with no volume change during the burning as it is the case for woody materials. Heat and mass transfer phenomena inside the solid are taken into account as well as the chemical reactions occurring in the condensed phase.

All processes are treated in one dimension corresponding to the axis of symmetry of the cone calorimeter.

Mass and species balance equations are solved. More details are given in [9-11].

The convective heat transfer coefficient of the back surface of the sample is considered as constant and equal to $0 \mathrm{~W} . \mathrm{m}^{-2} . \mathrm{K}$ for all the external heat fluxes considered. This corresponds to the boundary condition of an insulated surface.

The energy balance equation in the condensed phase is expressed as the following:

$$
\begin{aligned}
& \overline{\rho C_{p}} \frac{\partial T}{\partial t}=\frac{\partial}{\partial z}\left(k \frac{\partial T}{\partial z}\right)-\frac{\partial}{\partial z}\left(\bar{\varepsilon} \dot{q}_{0}^{\prime \prime} \exp (\bar{k} z)\right)-\sum_{i=1}^{i=n} \dot{w}_{k} \Delta H_{k} \\
& \dot{w}_{k}=\left(\frac{\bar{\rho} Y_{k} \Delta z}{\left(\bar{\rho} Y_{k} \Delta z\right)_{\Sigma}}\right)^{n_{k}} \frac{\left(\bar{\rho} Y_{k} \Delta z\right)_{\Sigma}}{\Delta z}\left(Y_{O_{2}}^{n_{O_{2, k}}}\right) A_{k} \exp \left(\frac{-E_{a_{k}}}{R T}\right)
\end{aligned}
$$

The full model including kinetic and thermal parts is unclosed due to the thermal properties of the condensed phase species that are unknown.

Thus, the model fitting method is first used to estimate the thermal properties of the different condensed phase species with the same method used before to estimate the kinetic parameters.

As for the kinetic parameters the mass loss rate given by the cone calorimeter experiments is used to find the set of thermal properties that best fits the curve. The estimation of these properties is made for one heat flux of $30 \mathrm{~kW} \cdot \mathrm{m}^{-2}$.

The heat flux of $20 \mathrm{~kW} \cdot \mathrm{m}^{-2}$ has not been taken into account to estimate the properties in order to avoid the problem of ignition that could occur for a too low heat flux.

Secondly, we used thermal properties as well as kinetic parameters estimated as inputs in GPYRO code to simulate the mass loss, the mass loss rate and temperature profiles given by the cone calorimeter experiments carried out at different heat fluxes.

Two heat fluxes have been simulated: the heat flux used to estimate properties and an additional heat flux of $50 \mathrm{~kW} \cdot \mathrm{m}^{-2}$ in order to validate the model. 
Numerical results have then been compared to experimental ones. The values of thermal properties estimated are presented in table 2 .

Table 2. Estimated thermal properties.

\begin{tabular}{|l|c|c|c|c|c|}
\hline Name & $\mathbf{k}\left(\mathbf{W} \cdot \mathbf{m}^{-\mathbf{2}} \cdot \mathbf{K}^{-\mathbf{1}}\right)$ & $\mathbf{\rho}\left(\mathbf{k g} \cdot \mathbf{m}^{-\mathbf{3}}\right)$ & $\mathbf{C}_{\mathbf{p}}\left(\mathbf{J}_{\mathbf{k}} \mathbf{k g}^{-\mathbf{1}} \cdot \mathbf{K}^{-\mathbf{1}}\right)$ & $\boldsymbol{\varepsilon}(-)$ & $\mathbf{\Upsilon}(-)$ \\
\hline plywood & 0.17 & 480 & 1700 & 0.90 & 0 \\
\hline$\alpha$ wood & 0.04 & 385 & 1850 & 0.872 & 0 \\
\hline$\beta$ wood & 0.03 & 378 & 1760 & 0.818 & 0 \\
\hline$\sigma$ wood & 0.07 & 63.8 & 1550 & 0.845 & 0 \\
\hline$\theta$ wood & 0.07 & 57.2 & 1720 & 0.92 & 0.26 \\
\hline$\lambda$ wood & 0.10 & 27 & 1950 & 0.95 & 0.693 \\
\hline
\end{tabular}

Figure 4 presents the comparison between experimental and calculated mass loss and mass loss rate as a function of time at an irradiance level of $30 \mathrm{~kW} \cdot \mathrm{m}^{-2}$. A good agreement between calculated and experimental results is obtained. The model developed here is also able to reproduce well the mass loss rate of the sample, which is one of the most important parameters in fire safety.
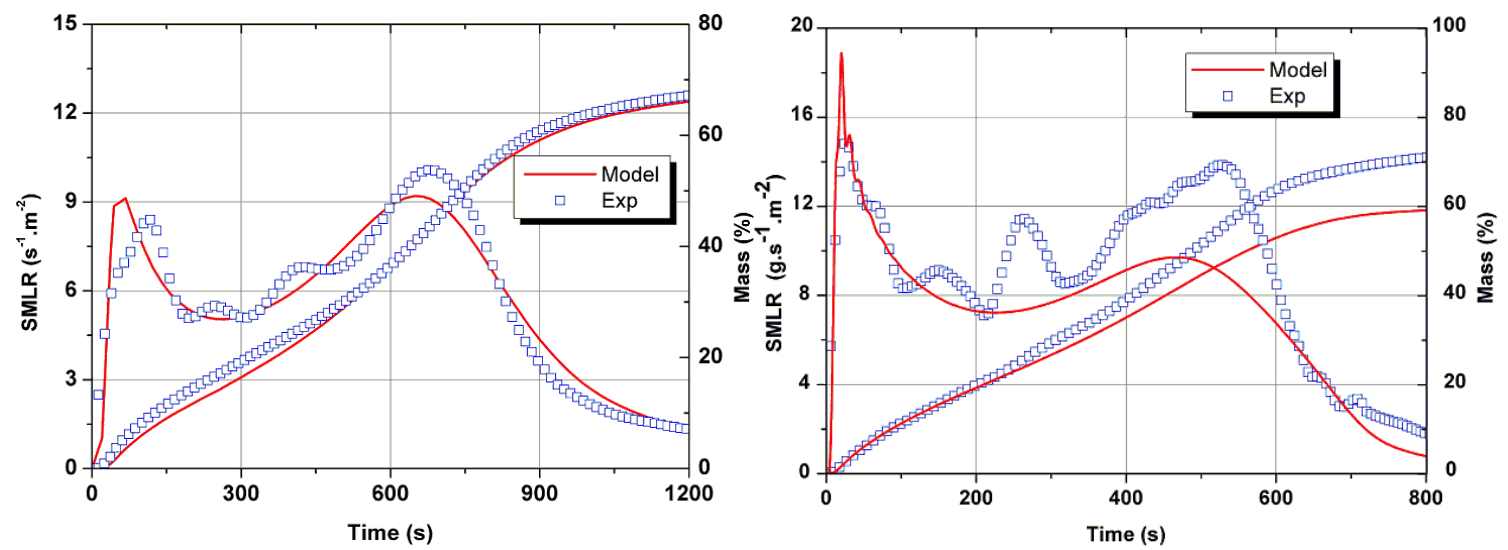

Fig. 4 Mass loss and mass loss rate evolutions as a function of time for 30 and $50 \mathrm{~kW} \cdot \mathrm{m}^{-2}$ respectively.
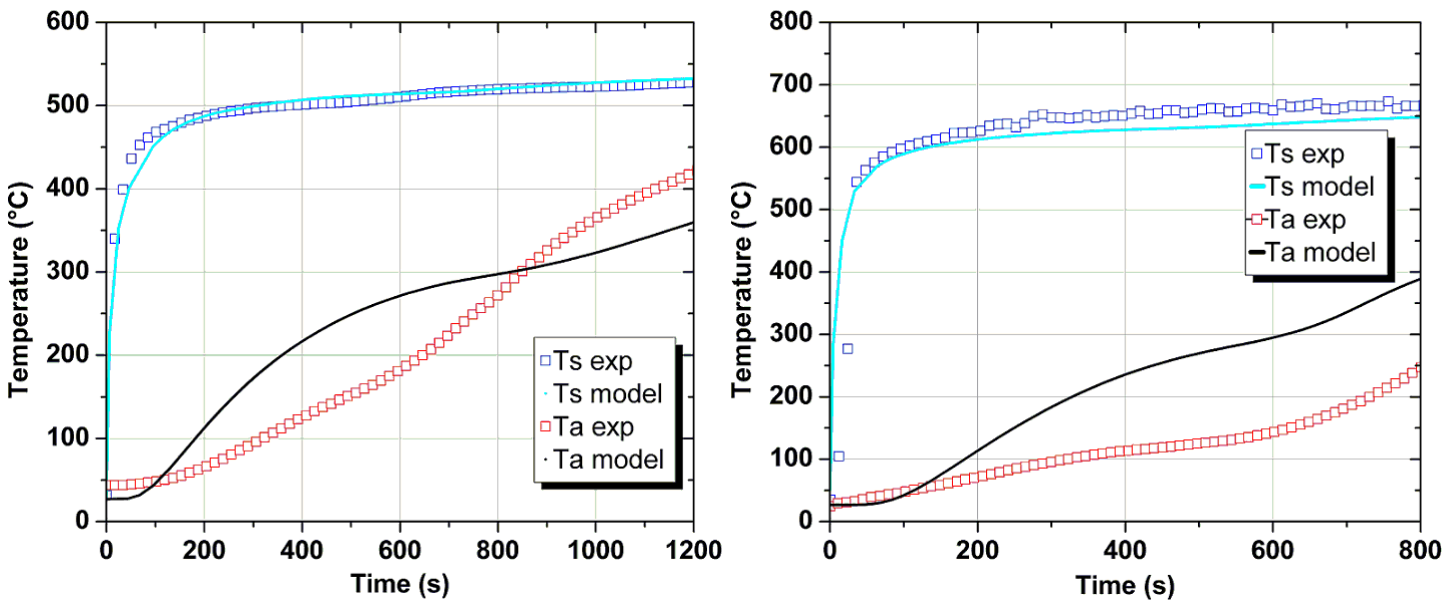

Fig. 5 Temperature evolutions of the front and back surfaces of the sample as a function of time for 30 and $50 \mathrm{~kW} \cdot \mathrm{m}^{-2}$ respectively. 
Figure 5 presents a comparison between experimental and calculated temperatures of the front surface of the sample directly exposed to the heat flux and the back surface of the sample located before the insulated layer during its decomposition in the cone calorimeter at $30 \mathrm{~kW} \cdot \mathrm{m}^{-2}$. The front and the back surfaces temperatures simulated show a general good agreement with the experimental data. However, we can notice that the back surface temperature is over predicted during the first $850 \mathrm{~s}$ and under predicted during the last 350s. This discrepancy is discussed further in comparison with the 3 steps mechanism.

The pyrolysis model developed here is rather complex because of its 5 reaction steps. A well-known simpler model has been tested under the same conditions in order to evaluate the influence of the complexity of the pyrolysis model on the prediction of the thermal decomposition of plywood. The second pyrolysis model proposed in [9-11] has been tested and can be written as follows:

$$
\left\{\begin{array}{l}
\text { wood } \rightarrow v_{1} \text { char }+v_{2} \text { thermal pyrolysate } \\
\text { wood }+v_{3} \mathrm{O}_{2} \rightarrow v_{4} \text { char }+v_{5} \text { oxydative pyrolysate } \\
\text { char }+v_{6} \mathrm{O}_{2} \rightarrow v_{7} \text { ash }+v_{8} \text { oxydative products }
\end{array}\right.
$$

The thermal properties and kinetic parameters estimated for this 3 steps mechanism for our case of plywood in cone calorimeter at $30 \mathrm{~kW} \cdot \mathrm{m}^{-2}$ are presented in Tables 3 and 4.

Table 3. Estimated thermal properties for the 3 steps model.

\begin{tabular}{|c|c|c|c|c|c|}
\hline Name & $K_{0}\left(W \cdot m^{-2} \cdot K^{-1}\right)$ & $\rho_{0}\left(\mathrm{~kg} \cdot \mathrm{m}^{-3}\right)$ & $C_{0}\left(J \cdot \mathrm{kg}^{-1} \cdot \mathrm{K}^{-1}\right)$ & $\varepsilon(-)$ & $r(-)$ \\
\hline wood & 0.18 & 580 & 1600 & 0.76 & 0 \\
\hline Char & 0.03 & 113 & 1260 & 0.98 & $4.82 \times 10^{-}$ \\
\hline ash & 0.08 & 13 & 1740 & 0.94 & 0.01 \\
\hline
\end{tabular}

Table 4. Estimated kinetic parameters for the 3 steps model.

\begin{tabular}{|c|c|c|c|c|c|c|c|}
\hline $\mathbf{K}$ & From & To & $\Delta H\left(\mathrm{~J}^{\prime} \mathrm{kg}^{-1}\right)$ & $\log _{10} A\left(s^{-1}\right)$ & $\mathrm{E}\left(\mathrm{J}^{\mathrm{mol}} \mathrm{mol}^{-1}\right)$ & $\mathbf{n}$ & $\mathbf{n}_{\mathrm{O} 2}$ \\
\hline 1 & wood & Char & $1.95 \times 10^{3}$ & 6.27 & 75.4 & 3.84 & 0 \\
\hline 2 & wood & Char & $-6.02 \times 10^{4}$ & 13.8 & 181 & 1.31 & 2.15 \\
\hline 3 & char & Ash & $-3.31 \times 10^{3}$ & 14 & 113 & 2.04 & 1.80 \\
\hline
\end{tabular}

Figure 6 presents a comparison of the experimental and predicted temperatures at the front and back surfaces of the solid during its decomposition in the cone calorimeter at $30 \mathrm{~kW} \cdot \mathrm{m}^{-2}$ for the 3 steps model. We can note that the temperatures of the front and back surfaces simulated show a general good agreement with the experimental data. The temperatures simulated with the 3 steps model are better than those simulated with the 5 steps model. The reason could be attributed to the enthalpy of volatilization estimated for the reactions of pyrolysis rather than the different condensed phase species produced for the two mechanisms. This point is discussed further. 


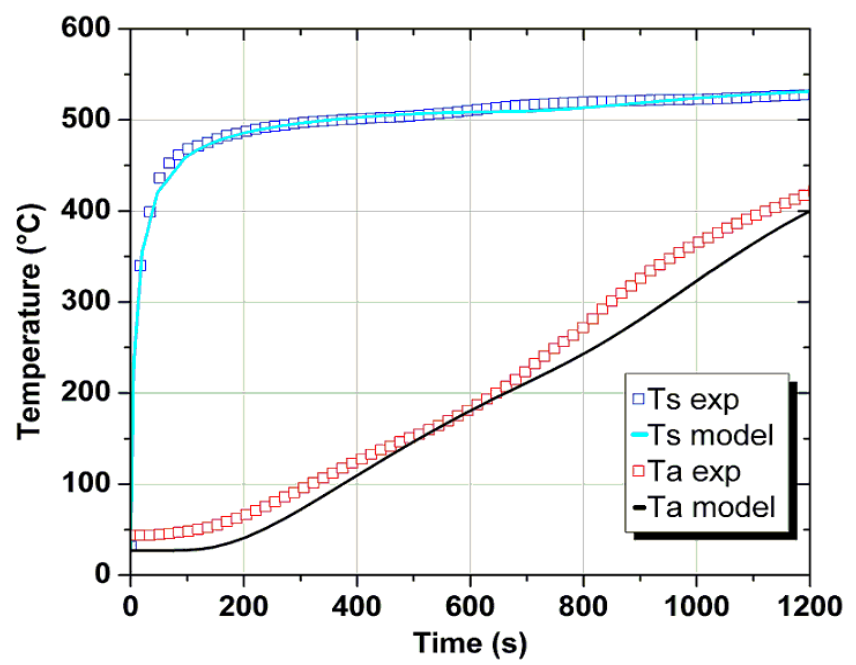

Fig. 6. Temperature evolutions as a function of time $\left(30 \mathrm{~kW} \cdot \mathrm{m}^{-2}\right)$ at the front and the back surface of the sample.

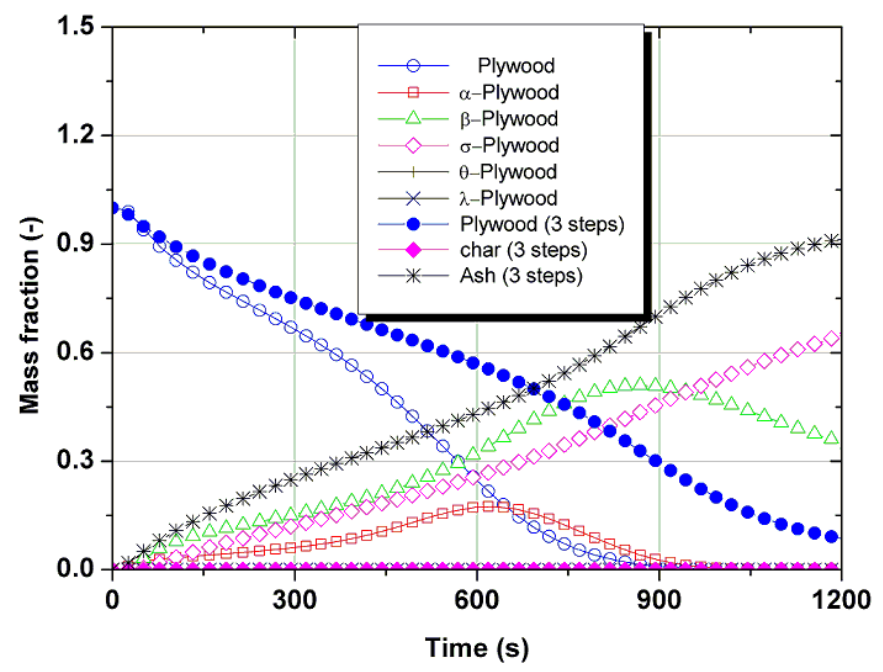

Fig 7: Numerical mass fractions of condensed species as function of time.

Figure 7 presents the evolution of the total (i.e. corresponding to the sum of mass fractions of each species along all the thickness of the sample) mass fractions of condensed phase species as a function of time. At 500 s the remaining plywood is $20 \% \mathrm{wt}$. lower for the 3 steps mechanism. At this time the mass fractions of charring species are also $20 \% \mathrm{wt}$. higher for the 5 steps mechanism than for the 3 steps mechanism. Since the thermal diffusivity of plywood is higher than those of the charring species, lower temperatures should be observed at the back surface for the 5 steps mechanism but it is not the case.

However, the enthalpy of volatilization for the 5 steps mechanism are lower (more than one order of magnitude) than those for the 3 steps mechanism. When these reactions are activated the energy consumed is less important for the 5 steps mechanism and could probably justify the over prediction of the back surface temperature.

Figure 8 presents the evolution of the mass loss and mass loss rate as a function of time at $30 \mathrm{~kW} . \mathrm{m}^{-2}$ for the model 3 steps. We can note a general good agreement with the experimental data except at the 
beginning of the test where the MLR is largely over predicted. The simulated MLR peak is reached at 20s. This discrepancy is discussed further by analyzing the mass fractions of condensed phase species.

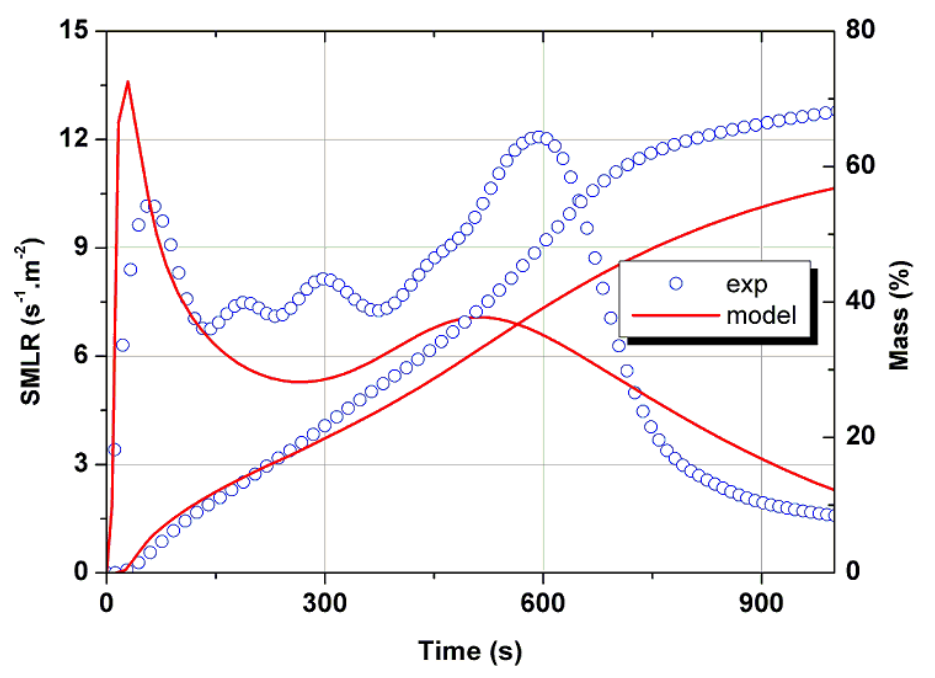

Fig. 8. Mass and mass loss rate evolutions as function of time at $30 \mathrm{~kW} \cdot \mathrm{m}^{-2}$.

Figure 9 presents the evolution of the mass fractions of condensed phase species and reaction rates of the first reaction of each mechanism as a function of the sample thickness at 20 s after start test. At this time plywood is decomposed over a thickness of $1 \mathrm{~mm}$ for the 2 models.

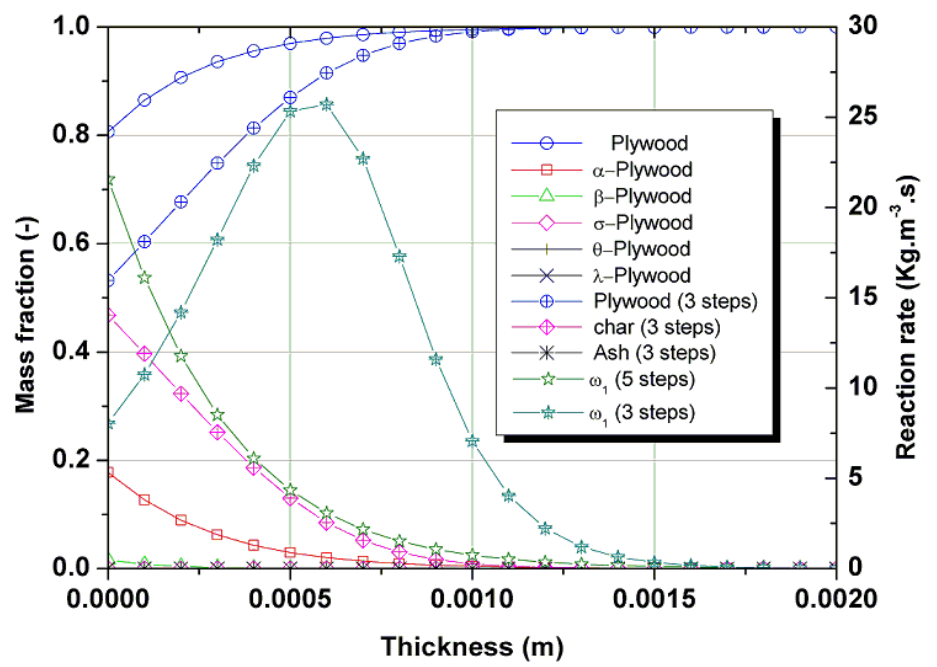

Fig. 9 Evolutions of mass fractions of condensed phase species as a function of time $\left(30 \mathrm{~kW} . \mathrm{m}^{-2}\right)$ at the back surface of the solid at 20 s.

We can note that more plywood is converted into condensed phase species (char) for the 3 steps model than for the 5 steps model. For the 3 steps model the production rate of char is higher than the production rate of the $\alpha_{\text {Plywood }}$ for the 5 steps model. Since the density of char is lower than the density of the virgin material (see estimated parameters in table 3 ) and no ash is produced at this time, the production of pyrolysis gases is high so is the mass loss rate (the density of condensed phase species appears in the reaction rates equations). 
Figure 10 presents the evolution of the mass fractions of the condensed phase species and the evolution of the temperature along the thickness of the sample at 1000 s for the 2 types of model. We can note that virgin plywood is present along all the thickness of the sample and represent $40 \% \mathrm{wt}$. of the sample weight at the back surface of the solid. However at this time no more virgin material remains for the corresponding experiment (see Fig 11).

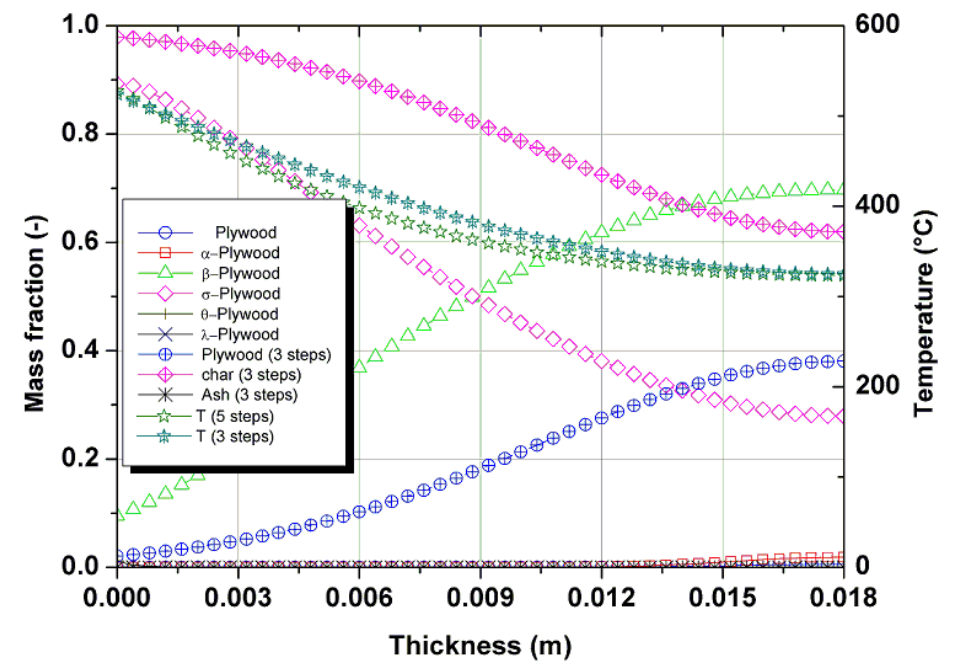

Fig. 10 Evolutions of mass fractions of condensed phase species as a function of time $\left(30 \mathrm{~kW} . \mathrm{m}^{-2}\right)$.at the back surface of the solid at $1000 \mathrm{~s}$.

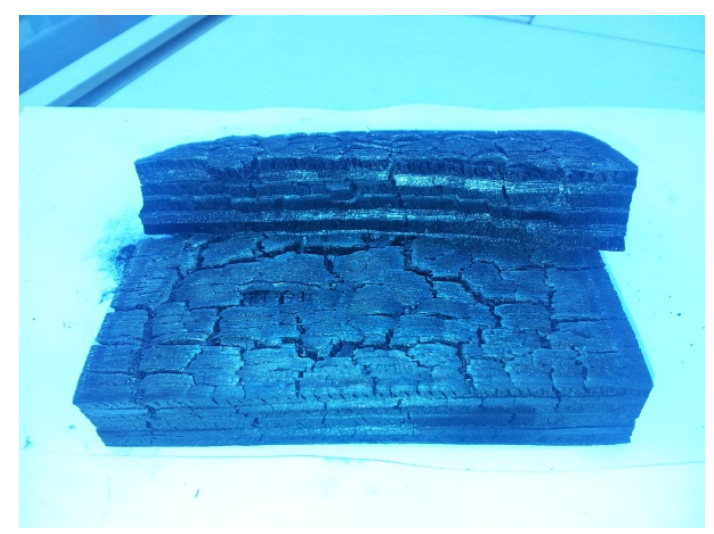

Fig. 11 Picture of the remaining solid at $1000 \mathrm{~s}$ under $30 \mathrm{~kW} \cdot \mathrm{m}^{-2}$.

Even the 3 steps pyrolysis model predicts quite well the mass loss rate of the plywood, its capacity to reproduce the thermal decomposition of the solid in depth is questionable. For the studied case, the 3 steps pyrolysis model gives some non-physical results.

\section{CONCLUSION}

The thermal decomposition of a plywood sample has been investigated using a multi-scale methodology. In TGA scale, five heating rates and two conditions of atmosphere have been chosen to conduct experiments. The results allowed to propose and to validate a kinetic mechanism ( 5 steps) of the thermal decomposition of the plywood. The kinetic parameters were estimated using a model fitting method: the genetic algorithms technique. The kinetic mechanism involving 5 steps has been established by analyzing the mass 
loss rate curves as well the gases released. The kinetic parameters of the reactions identified have also been estimated.

Some experiments have been conducted in the cone calorimeter in order to take into account heat and mass transfer phenomena into the sample. A model describing heat and mass transfer phenomena has been added to the kinetic part by using the GPYRO program. The unknown thermal properties of condensed phase species have been estimated using a model fitting method: the genetic algorithms.

The development of the full pyrolysis model has been realized for a heat flux of $30 \mathrm{~kW} . \mathrm{m}^{-2}$. A full simulation has been conducted for a heat flux of $50 \mathrm{~kW} \cdot \mathrm{m}^{-2}$ in order to validate the model. Only the temperature of the back surface of the sample is not correctly predicted. The temperature of the front surface of the sample, the mass loss and mass loss rate are quite well predicted.

In order to examine the influence of the complexity of the kinetic mechanism on the description of the thermal decomposition, a simpler kinetic mechanism has been investigated. The results have been compared to those given by the more complex one ( 5 steps).

The 3 steps mechanism predicted quite well the temperatures of the front and back surfaces of the sample but some discrepancies have been observed for the mass loss rate prediction. The major point concerns the capacity of the 3 steps mechanism to reproduce the evolution of the mass fractions of the condensed phase species. Indeed some unphysical results have been observed mainly concerning the evolution of the virgin material as a function of time. It has been showed that the 3 steps mechanism predicted a fraction of remaining plywood at the end of the test which was not observed in the experiment.

This work shows that when the validation of a pyrolysis model is carried out with respect to more than one parameter (i.e. not only MLR), a complex kinetic mechanism must be considered.

\section{Acknowledgments}

This work pertains to the French Government program "Investissements d'Avenir" (LABEX INTERACTIFS, reference ANR-11-LABX-0017-01).

\section{REFERENCES}

[1] Jiang, Y., (2006) decomposition, Ignition and Flame Spread on Furnishing Materials, Ph.D. Thesis, Victoria University. Australia.

[2] Branca, C., and Di Blasi, C., (2004) Global intrinsic kinetics of wood oxidation, Fuel 83: 81-87.

[3] Wilkie, C.A., (1999) TGA-FTIR an extremely useful technique for studying polymer degradation, Polymer Degradation and Stability 66: 301-306.

[4] Di Blasi, C., (2008) Modeling chemical and physical processes of wood and biomass pyrolysis, Progress in Energy and Combustion Sciences 34: 47-90.

[5] Chuang, F.S., (2007) Analysis of thermal degradation of diacetylene-containing polyurethane copolymers, Polymer Degradation and Stability 92: 1393-1407.

[6] Richard, W.C., and Mark A.S., (2009) Quantitative flash pyrolysis Fourier transforms infrared spectroscopy of organic materials," Analytica Chimica Acta 639: 62-66.

[7] Fang, M.X., Shen, D.K., Li, Y.X., Yu, C.J., Luo, Z.Y., and Cen, K.F., (2006) Kinetic study on pyrolysis and combustion of wood under different oxygen concentrations by using TG-FTIR analysis, Journal of Analytical and Applied Pyrolysis 77: 22-27.

[8] ISO 5660 (1993)., Fire test Reaction to fire - Rate of heat release from building products, ISO, Geneva. 
[9] Lautenberger, C., and Fernandez-Pello, C., (2009) A model for the oxidative pyrolysis of wood, Combustion and Flame 156: 1503-1513.

[10] Lautenberger, C., and Fernandez-Pello, C., (2009) Generalized pyrolysis model for combustible solids, Fire Safety Journal 44: 819-839.

[11] Lautenberger, C., (2007) A generalized pyrolysis model for combustible solids. Ph.D Thesis, Department of Mechanical Engineering, University of California, Berkeley.

[12] Fateh, T., Rogaume, T., Luche, J., Richard, F., Jabouille, F., (2013) Modeling of the thermal decomposition of a treated plywood from thermo-gravimetry and Fourier-transformed infrared spectroscopy experimental analysis, Journal of Analytical and Applied Pyrolysis 101: 35-44..

[13] Reeves, C.R., and Rowe, J.E., (2002) Genetic algorithms principles and perspective, Computer Science Interfaces Series 20: 1-340.

[14] Matala, A., Estimation of solid phase reaction parameters for fire simulation, A dissertation for the degree of master of science in technology in the degree program in engineering physics, University of Technology, Helsinki. (2008).

[15] Lautenberger, C., and Fernandez-Pello, C., (2006) The application of a Genetic algorithm to estimate material properties for fire modeling from bench scale Fire test data, Fire Safety Journal 41: 204-214.

[16] Rein, G., Lautenberger, C., Fernandez-Pello, C., Torero, J., and Urban, D., (2006) Application of genetic algorithms and thermogravimetry to determine the kinetics of polyurethane foam in smoldering combustion, Combustion and Flame 146: 95-108.

[17] Torero, J.L., "Flaming ignition of solid fuels," The SFPE Handbook of Fire protection engineering (4 ${ }^{\text {nd }}$ ed), DiNenno P.J. (ed.), National Fire Protection Association, Quincy, MA 02269, 2008, p. 2/262.

[18] Hirata, T., Kawamoto,S., and Nishimoto, T., (1991) Thermogravimetry of wood treated with water-soluble retardants and a proposal for development of fire retarded wood materials, Fire and Materials 15: 27-36.

[19] Mehrabian, R., Scharler, R., and Obernberger, I., (2012) Effects of pyrolysis conditions on the heating rate in biomass particles and applicability of TGA kinetic parameters in particle thermal conversion modeling, Fuel 93: 567-575. 